
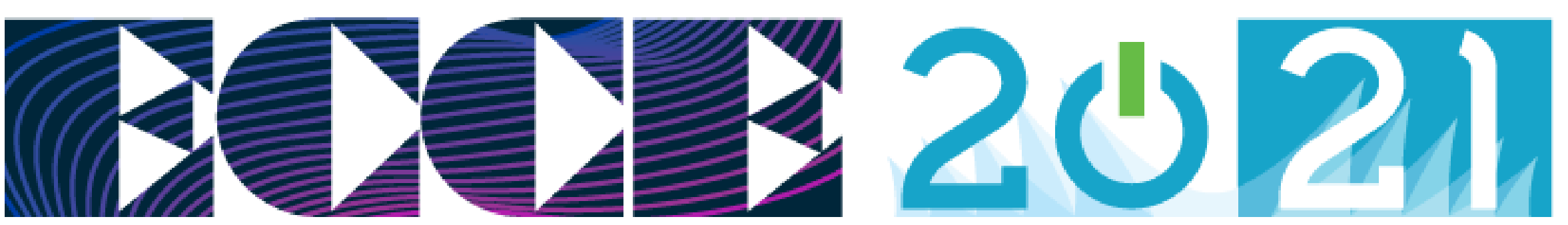

IEEE ENERGY CONVERSION CONGRESS \& EXPO Vancouver, Canada $\cong O c t .10-14$

\title{
Design Power Control Strategies of Grid-Forming Inverters for Microgrid Application
}

\author{
Jing Wang, Senior Research Engineer \\ National Renewable Energy Laboratory \\ $10 / 12 / 2021$
}




\section{Background}

- State-of-the-art grid-forming inverter control: PQ in grid-connected (current source) and VF in islanded mode (voltage source)

- Problem: phase jump during microgrid transition operation

- Solution: use grid-forming control in both grid-connected and islanded mode

- Problem: grid-forming control controls system voltage rather than power.

- Objective: design power control strategy of grid-forming inverters for microgrid applications How to control PQ via grid-forming control in grid-connected mode

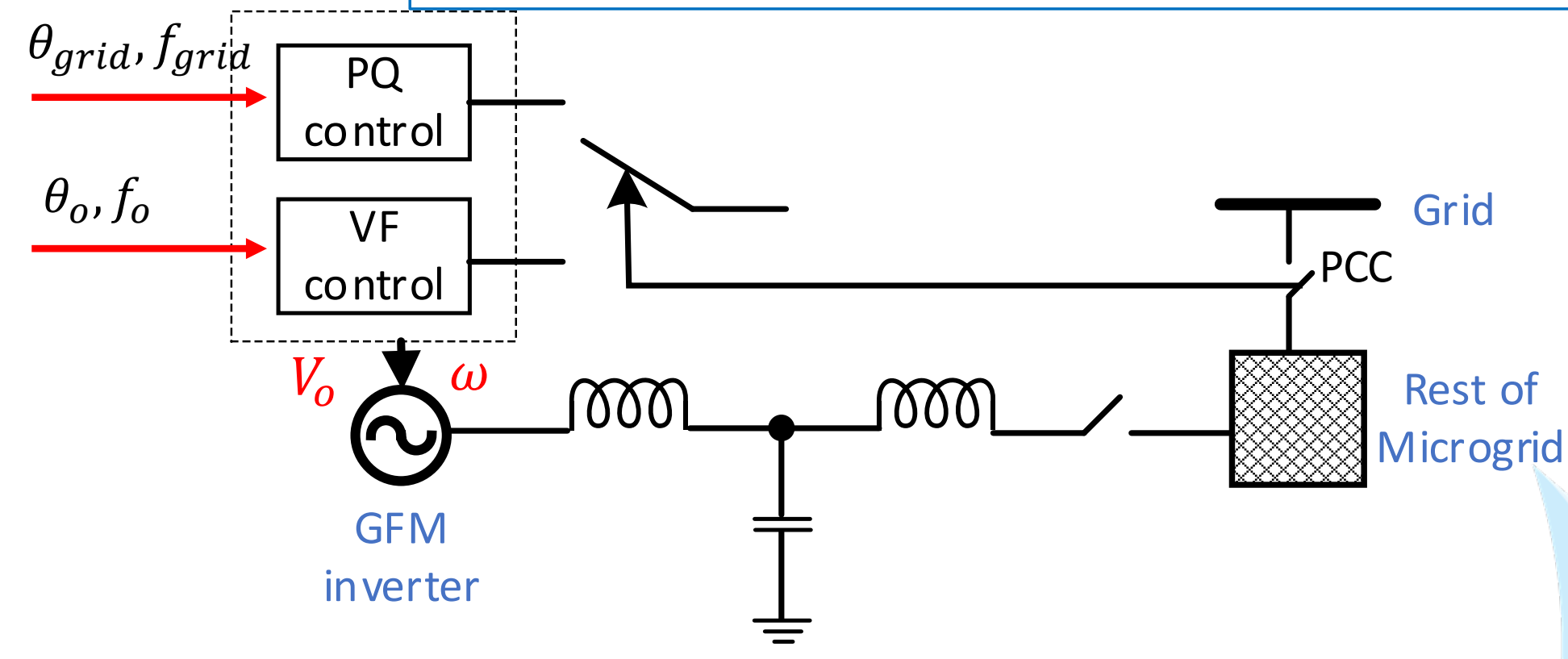




\section{Solution}

- Strategy I: Current controller outer loop in grid-connected, and droop control in islanded mode

- Strategy II: Droop control in both grid-connected and islanded mode
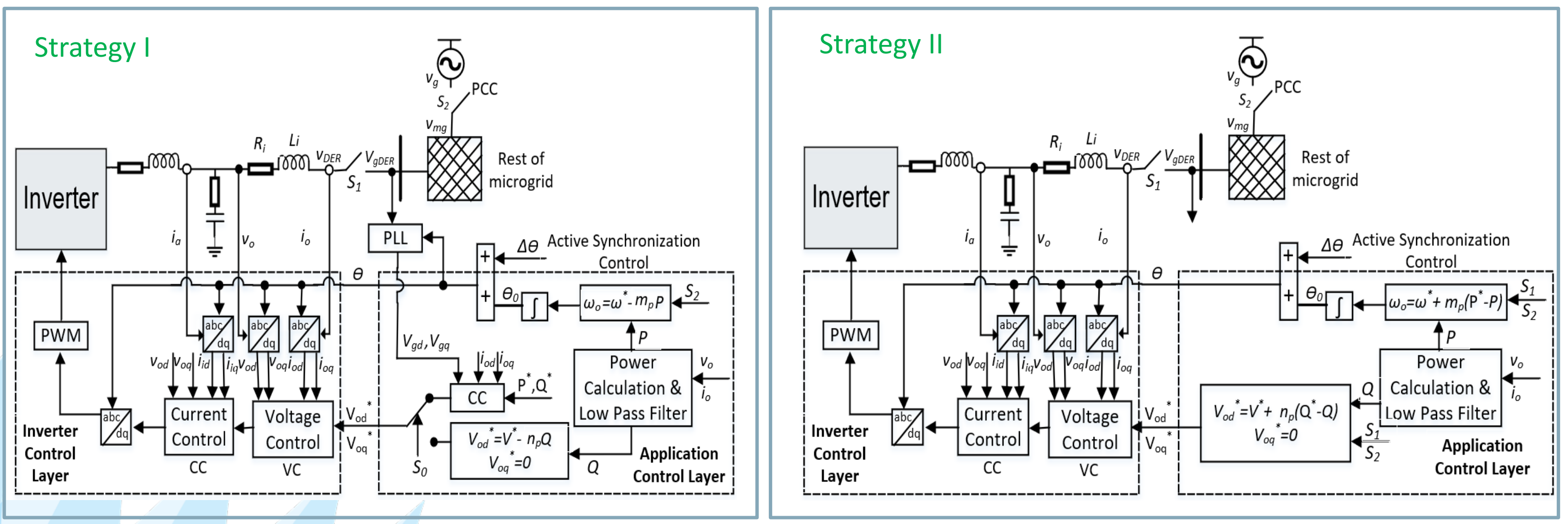


\section{Comparison of These Two Strategies}

\begin{tabular}{|c|c|c|}
\hline \begin{tabular}{|l|} 
Control \\
Strategy \\
\end{tabular} & Startup & \\
\hline Strategy I & $\begin{array}{c}\omega_{o}=\omega^{*} \\
V_{o d}^{*}=V^{*} \\
V_{o q}^{*}=0\end{array}$ & $\begin{array}{l}V_{o d}^{*}=V_{g d}+R_{i} i_{o d}^{*}+K_{p} \\
V_{o q}^{*}=V_{g q}+R_{i} i_{o q}^{*}+K_{p o}\end{array}$ \\
\hline Strategy II & $\begin{array}{c}\omega_{o}=\omega^{*} \\
V_{o d}^{*}=V^{*} \\
V_{o q}^{*}=0\end{array}$ & $\begin{array}{r}\omega_{o}=\omega^{*}+m_{p} \\
V_{o d}^{*}=V^{*}+n_{p}\left(Q^{*}-Q\right)\end{array}$ \\
\hline $\begin{array}{l}i_{o d} \\
i_{o q}\end{array}$ & $\begin{array}{l}\left(V_{o d}-V_{g}\right. \\
\left(V_{o q}-V_{g c}\right.\end{array}$ & $\begin{array}{l}\left.\omega_{o} L_{i} i_{o q}\right), P=\frac{3}{2} V_{g d} i_{o d} \\
\left.\omega_{o} L_{i} i_{o d}\right), Q=-\frac{3}{2} V_{o g} i_{o q}\end{array}$ \\
\hline
\end{tabular}

Strategy l: decouple the intrinsic power-angle characteristics
Grid-Connected $\quad$ Islanded

$$
\omega_{o}=\omega^{*}
$$

$\omega_{o}=\omega^{*}-m_{p} P$

$V_{o d}^{*}=V^{*}-n_{p} Q$

$$
V_{o q}^{*}=0
$$

$\omega_{o}=\omega^{*}-m_{p} P$

$V_{o d}^{*}=V^{*}-n_{p} Q$

$V_{o q}^{*}=0$

$$
V_{o q}^{*}=0
$$



Strategy II: follow the power-angle characteristics
J Wang, $B$. Lundstrom, A.

Bernstein,

"Design of a nonpll grid-forming inverter for smooth microgrid transition operation," IEEE PESGM 2020.

Different power-angle stability 


\section{Comparison of These Two Strategies}

\begin{tabular}{|c|c|c|c|}
\hline $\begin{array}{l}\text { Control } \\
\text { Strategy }\end{array}$ & Startup & Grid-Connected & Islanded \\
\hline Strategy I & $\begin{array}{c}\omega_{o}=\omega^{*} \\
V_{o d}^{*}=V^{*} \\
V_{o q}^{*}=0\end{array}$ & $\begin{array}{c}\omega_{o}=\omega^{*} \\
V_{o d}^{*}=V_{g d}+R_{i} i_{o d}^{*}+K_{p o}\left(i_{o d}^{*}-i_{o d}\right)-\omega_{o} L_{i} i_{o q}, i_{o d}^{*}=\frac{2}{3} \frac{P^{*}}{V_{g d}} \\
V_{o q}^{*}=V_{g q}+R_{i} i_{o q}^{*}+K_{p o}\left(i_{o q}^{*}-i_{o q}\right)+\omega_{o} L_{i} i_{o d}, i_{o q}^{*}=-\frac{2}{3} \frac{Q^{*}}{V_{g d}}\end{array}$ & $\begin{array}{l}\omega_{o}=\omega^{*}-m_{p} P \\
V_{o d}^{*}=V^{*}-n_{p} Q \\
V_{o q}^{*}=0\end{array}$ \\
\hline Strategy II & $\begin{aligned} \omega_{o} & =\omega^{*} \\
V_{o d}^{*} & =V^{*} \\
V_{o q}^{*} & =0\end{aligned}$ & $\begin{array}{c}\omega_{o}=\omega^{*}+m_{p}\left(P^{*}-P\right) \rightarrow \omega_{o}=\omega^{*}, P^{*}=P \\
V_{o d}^{*}=V^{*}+n_{p}\left(Q^{*}-Q\right) \rightarrow V_{o d}^{*}=V^{*}+\left(n_{p}+\frac{k p}{s}\right)\left(Q^{*}-Q\right)\end{array}$ & $\begin{array}{c}\omega_{o}=\omega^{*}-m_{p} P \\
V_{o d}^{*}=V^{*}-n_{p} Q \\
V_{o q}^{*}=0\end{array}$ \\
\hline
\end{tabular}

\section{Strategy I: $\omega^{*}-m_{p} \mathrm{P} \rightarrow \omega^{*}, v_{o d}^{*}, v_{o q}^{*}$ has big step changes}

Strategy II: $\omega^{*}-m_{p} \mathrm{P} \rightarrow \omega^{*}+\Delta \omega, v_{o d}^{*}$ has small step change

$v_{o q}^{*}=0$ all the time without change

- Both strategies are expected to have transients

- Strategy I should have better transients $\omega^{*} \rightarrow \omega^{*}-m_{p} P$ for both strategies

Strategy I: $v_{o d}^{*}, v_{o q}^{*}$ has big step changes Strategy II: $v_{o d}^{*}$ has small step change $v_{o q}^{*}=0$ all the time without change

Strategy II should have better transients 


\section{Comparison of These Two Strategies}

Generated using the GFM inverter angle instead of its own angle

\begin{tabular}{|c|c|c|}
\hline Control Strategy & Grid-Connected & Power Tracking Performance \\
\hline Strategy I & $\begin{array}{c}\omega_{o}=\omega^{*} \\
V_{o d}^{*}=V_{g d}+R_{i} i_{o d}^{*}+K_{p o}\left(i_{o d}^{*}-i_{o d}\right)-\omega_{o} L_{i} i_{o q}, i_{o d}^{*}=\frac{2}{3} \frac{P^{*}}{V_{g d}} \\
V_{o q}^{*}=V_{g q}+R_{i} i_{o q}^{*}+K_{p o}\left(i_{o q}^{*}-i_{o q}\right)+\omega_{o} L_{i} i_{o d}, i_{o q}^{*}=-\frac{2}{3} \frac{Q^{*}}{V_{g d}}\end{array}$ & $\begin{array}{l}\qquad V_{g d} \approx V_{g d}^{\prime}, V_{g q} \neq V_{g q}^{\prime}=0 \\
\text { - Active power tracking can be } \\
\text { acceptable } \\
\text { - Reactive power tracking has } \\
\text { discrepancy }\end{array}$ \\
\hline $\begin{array}{r}\text { Strategy II } \\
\text { V }\end{array}$ & $\begin{array}{c}\omega_{o}=\omega^{*}+m_{p}\left(P^{*}-P\right) \rightarrow \omega_{o}=\omega^{*}, P^{*}=P \\
V_{o d}^{*}=V^{*}+n_{p}\left(Q^{*}-Q\right) \rightarrow V_{o d}^{*}=V^{*}+\left(n_{p}+\frac{k_{n p}}{s}\right)\left(Q^{*}-Q\right) \\
V_{o q}^{*}=0\end{array}$ & $\begin{array}{l}\text { - Active power must track } P^{*} \text { to } \\
\text { reach steady-state frequency for } \\
\text { stability } \\
\text { - Reactive power can track the } Q^{*} \\
\text { because of PI control }\end{array}$ \\
\hline
\end{tabular}

Strategy I: terminal voltage dependent

Strategy II: less dependent on the terminal voltage

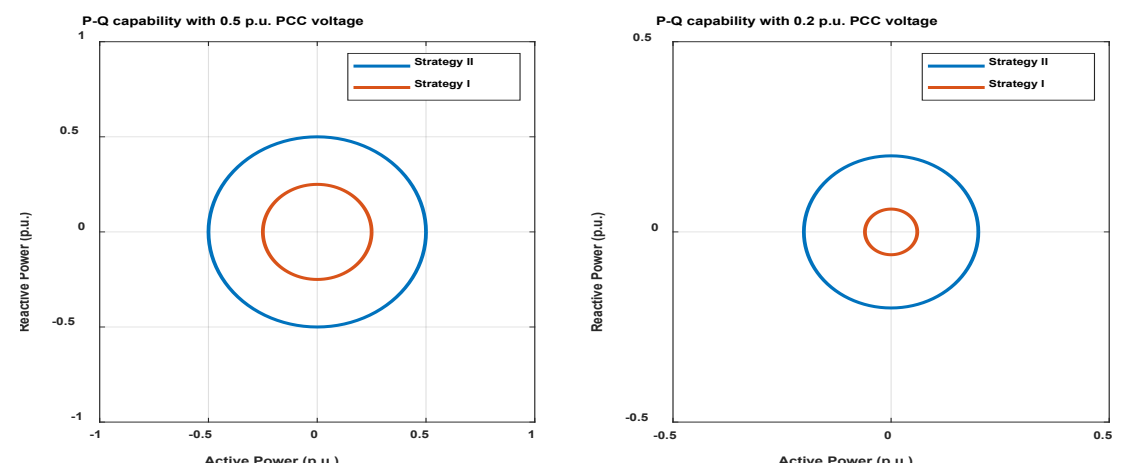




\section{Simulation Results}

Unplanned islanding operation

- Microgrid: two GFM battery inverters, PVs grid-following, residential and commerical loads

- Simulation software: Matlab Simulink

- Events: grid-connected, unplanned isInding at $10 \mathrm{~s}$, planned reconnection at $15 \mathrm{~s}$, reconnect to the grid.

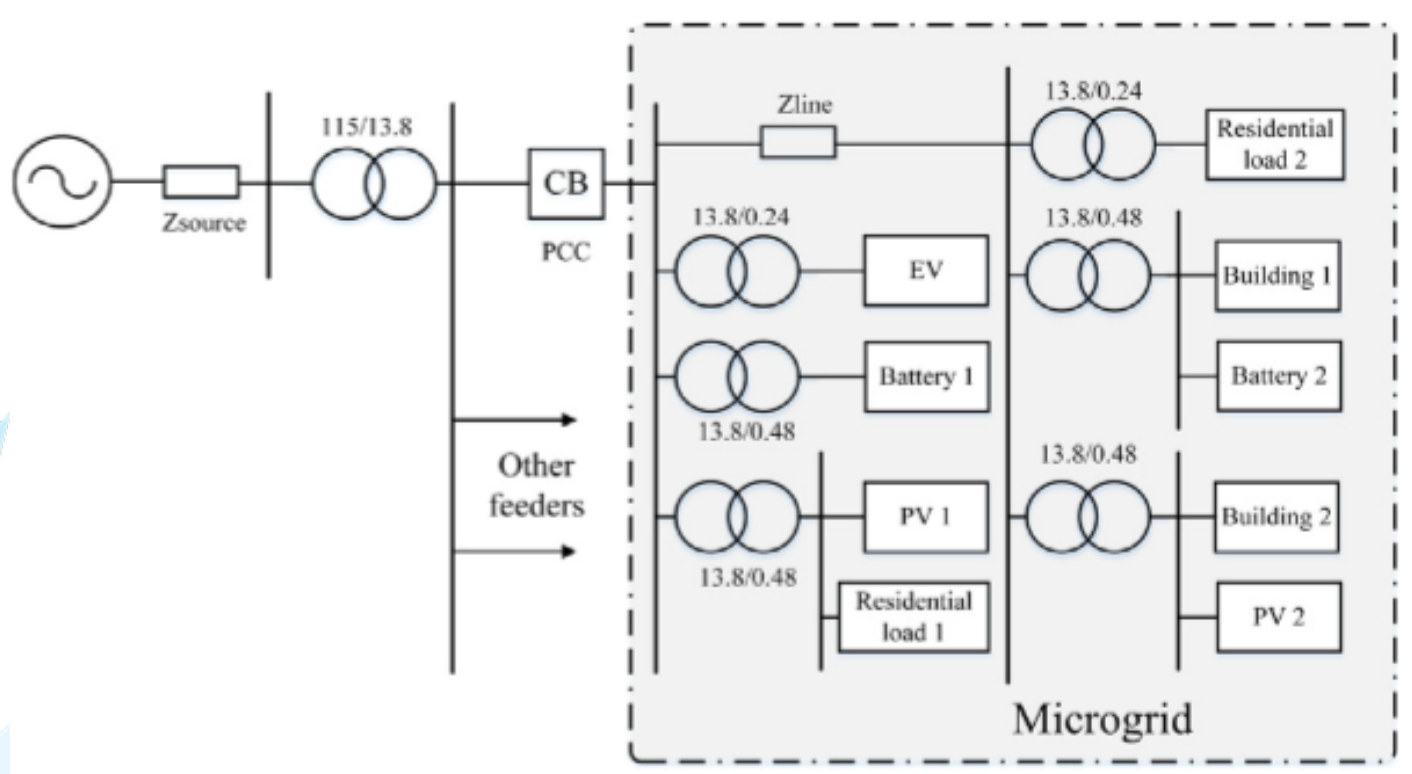

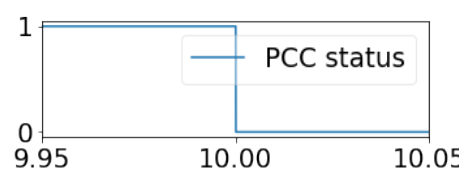
10.05

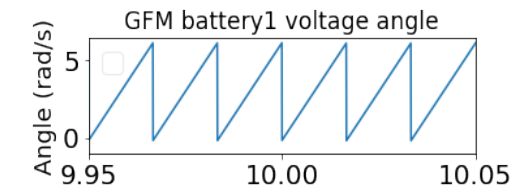

GFM batteryl voltage


$$
\text { 安 } 9.95
$$


GFM battery2 voltage


Strategy I

IEEE ENERGY CONVERSION CONGRESS \& EXPO

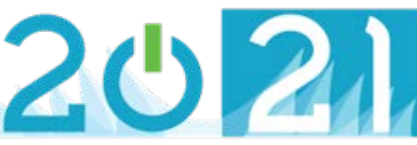

Vancouver, Canada $\cong$ Oct. 10-14

gy 


\section{Simulation Results - Reconnection to the Grid}

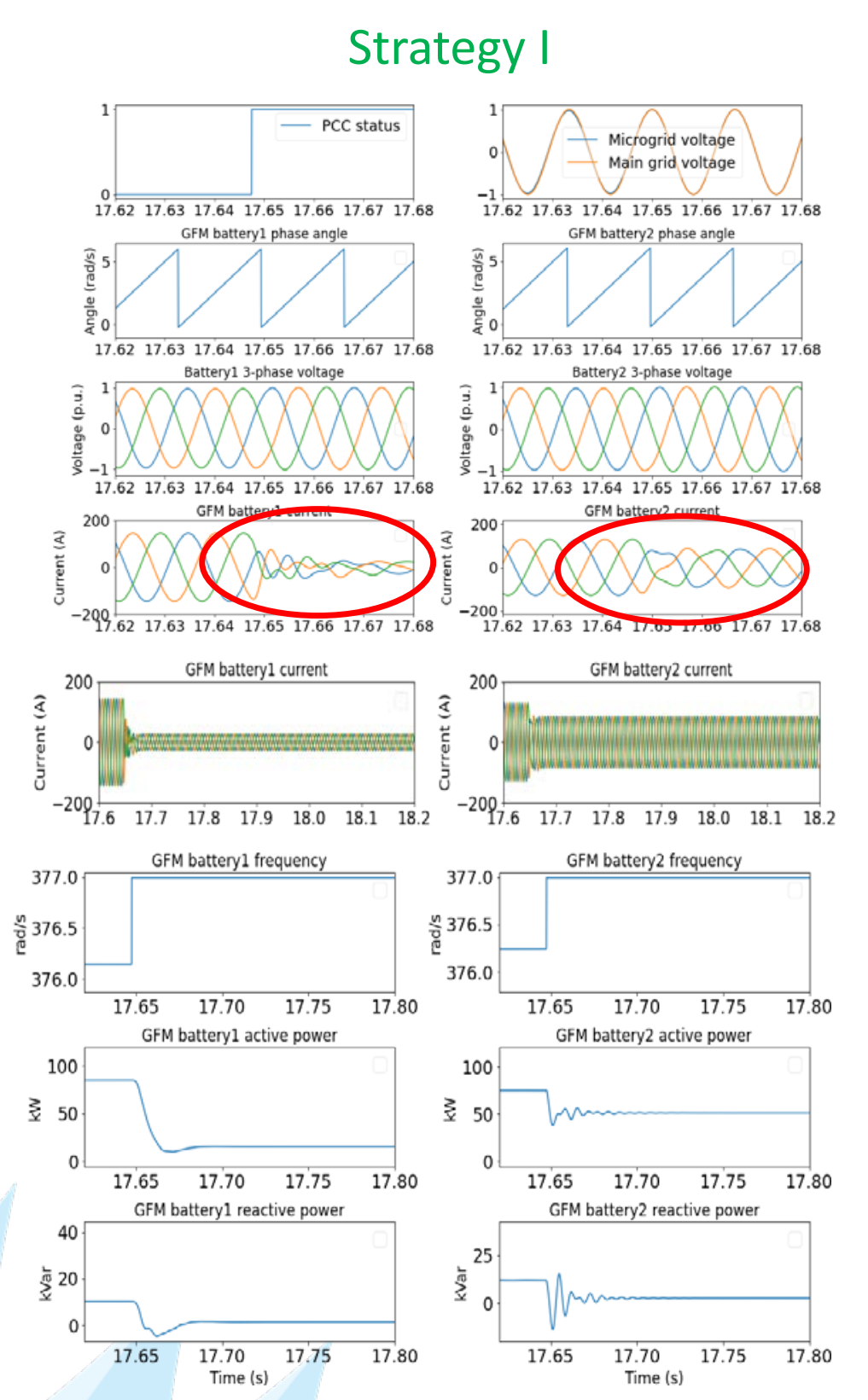

Strategy II
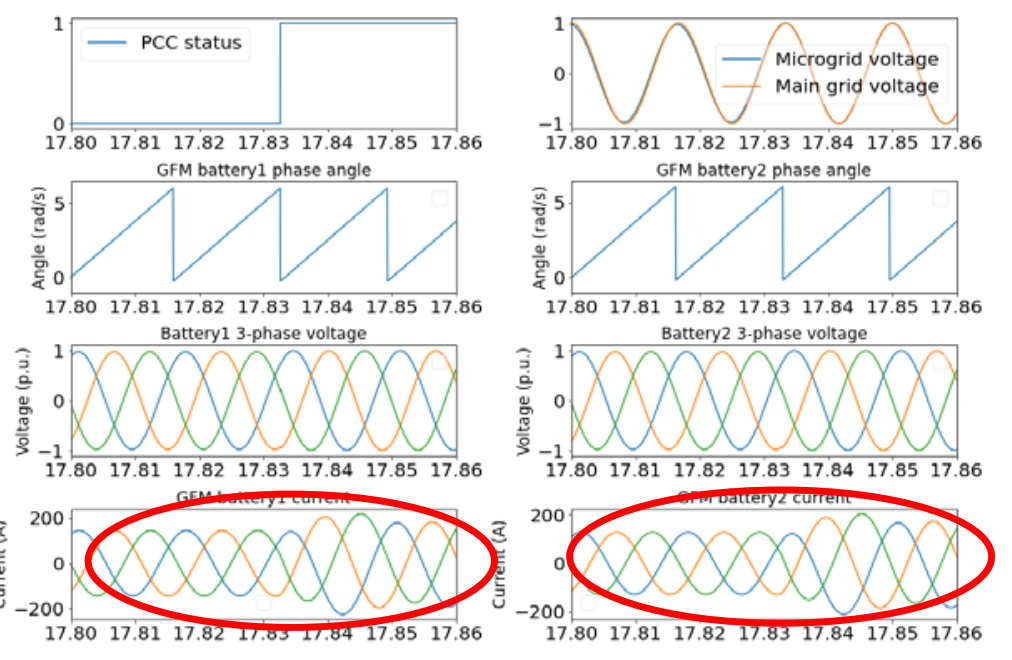

1 Battery2 3.-phase voltage


GFM battery2 current
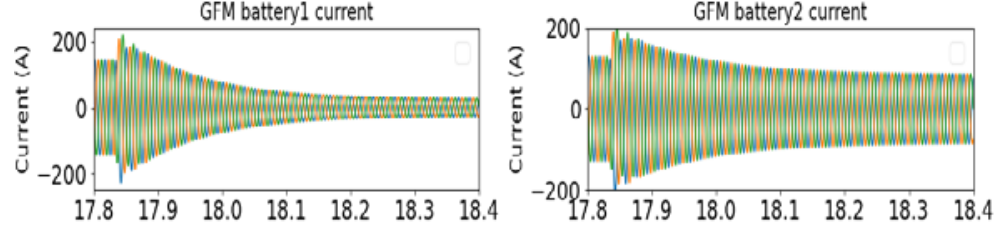

GFM batteryl frequency
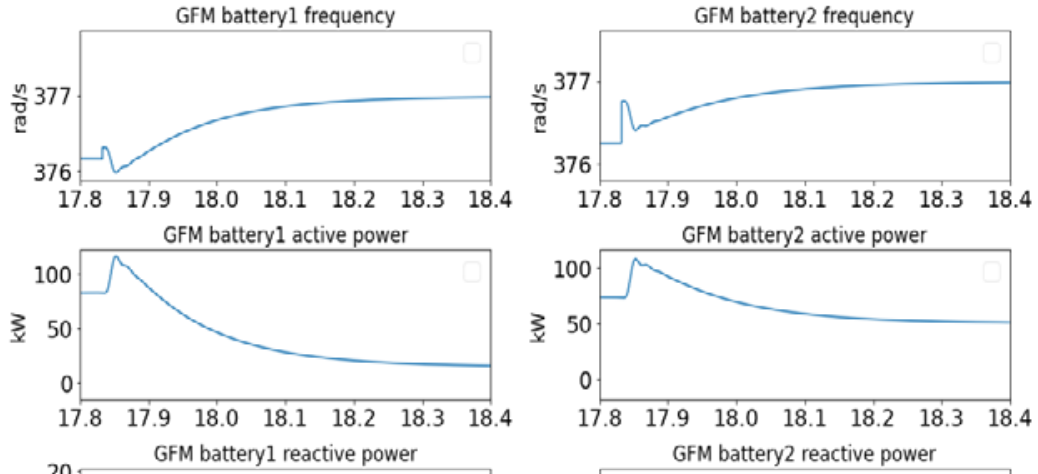



GFM battery1 reactive power

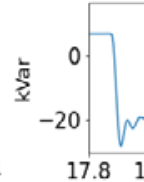




\section{Simulation Results}

- Power tracking performance in gridconnected mode


- P-Q capability with low PCC voltages in grid-connected mode Strategy I GFM inverter voltage (p.u.)


Strategy I GFM inverter current (p.u.)



\begin{tabular}{llllll}
\hline 98 & 12.00 & 12.02 & 12.04 & 12.06 & 12.08
\end{tabular}

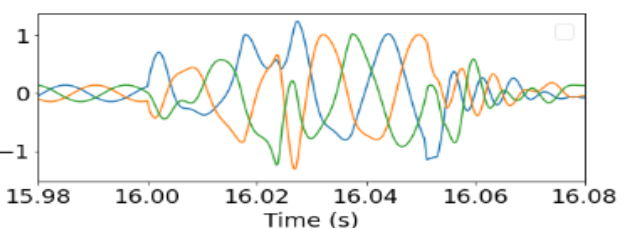

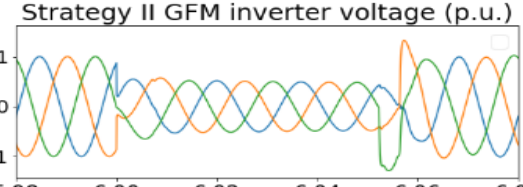
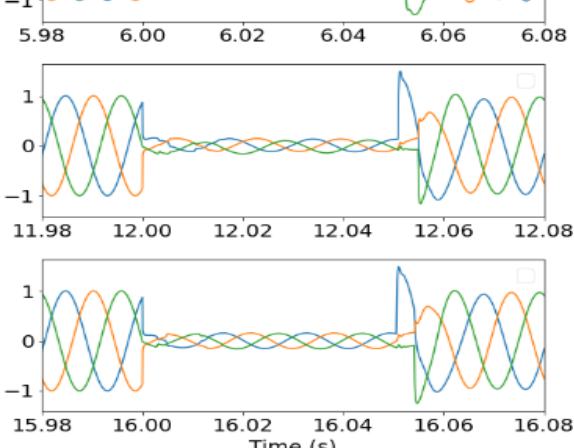

Strategy II GFM inverter current (p.u.)

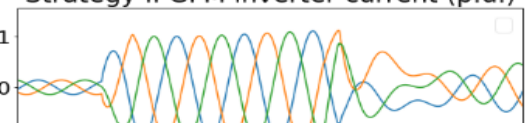

\begin{tabular}{lllllll}
-1 & \multicolumn{7}{c}{ 5.98 } & 6.00 & 6.02 & 6.04 & 6.06 & 6.08 & 6.10
\end{tabular}

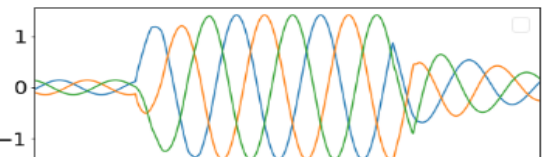

$\begin{array}{llllll}11.98 & 12.00 & 12.02 & 12.04 & 12.06 & 12.0\end{array}$

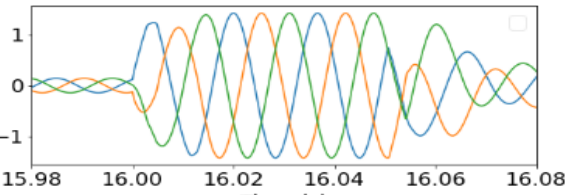

$\begin{array}{llllll}15.98 & 16.00 & 16.02 & 16.04 & 16.06 & 16.08\end{array}$ 000

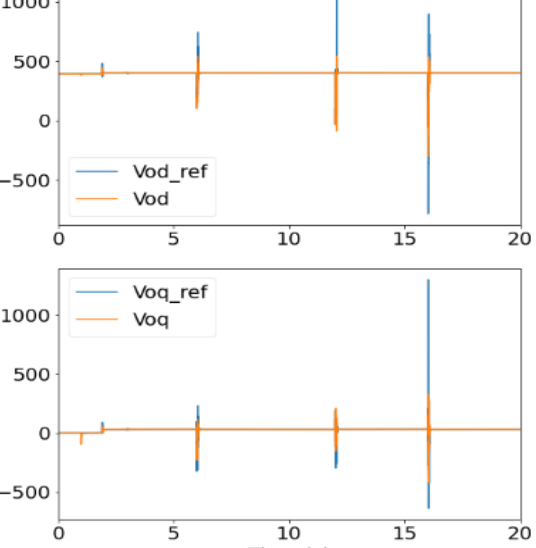

Strategy II GFM inverter Voltage in do

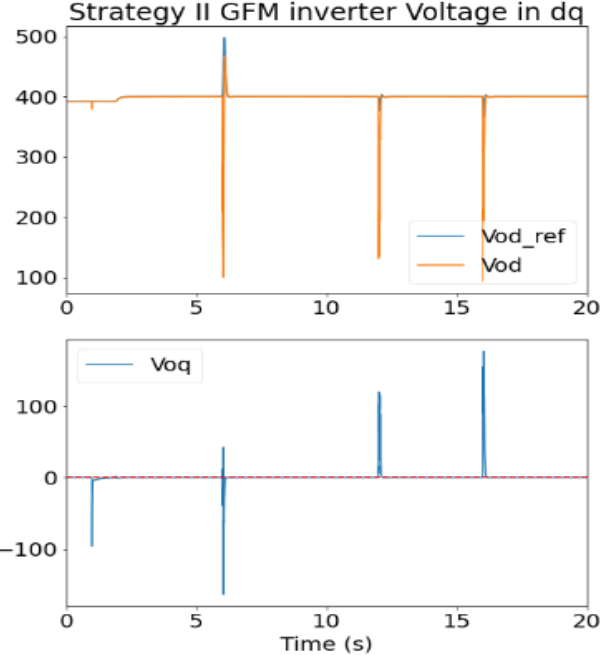




\section{Conclusion}

\begin{tabular}{|l|l|}
\hline \multicolumn{1}{|c|}{ Circumstance } & \multicolumn{1}{c|}{ Strategy Comparison } \\
\hline Unplanned islanding & $\begin{array}{l}\text { Both have smooth transients. Strategy II has slightly } \\
\text { better transients in the output current. }\end{array}$ \\
\hline $\begin{array}{l}\text { Reconnection } \\
\text { operation }\end{array}$ & $\begin{array}{l}\text { Strategy I has better transients in frequency, output } \\
\text { current, and power. }\end{array}$ \\
\hline Power tracking & $\begin{array}{l}\text { Strategy I reaches steady state faster with overshoots } \\
\text { and has a tracking error in the reactive power. } \\
\text { Strategy II has good tracking performance for both } \\
\text { active and reactive power with an acceptable settling } \\
\text { time. }\end{array}$ \\
\hline $\begin{array}{l}\text { P-Q capability with } \\
\text { low PCC voltage }\end{array}$ & $\begin{array}{l}\text { The low PCC voltage has a larger impact for Strategy } \\
\text { I because its power control loop is a current control } \\
\text { loop, and the current references depend on the PCC } \\
\text { voltage. Strategy II has a larger P-Q capability with } \\
\text { low PCC voltages and can maintain stability during } \\
\text { fault ride-through. Strategy I can maintain stability } \\
\text { only when the voltage is not less than a certain level. }\end{array}$ \\
\hline
\end{tabular}

- Overall, Strategy II is recommended for microgrid applications

- Comparable transition operation performance

- Better power tracking in grid-connected mode

- Better P-Q capability under faults or low PCC voltages

- Easy for implementation.

This work was authored by the National Renewable Energy Laboratory, operated by Alliance for Sustainable Energy, LLC, for the U.S. Department of Energy (DOE) under Contract No. DE-AC3608GO28308. Funding was provided by the U.S. DOE's Solar Technology Energy Office (SETO) program. The views expressed in the article do not necessarily represent the views of the DOE or the U.S. Government. The U.S. Government retains and the publisher, by accepting the article for publication, acknowledges that the U.S. Government retains a nonexclusive, paid-up, irrevocable, worldwide license to publish or reproduce the published form of this work, or allow others to do so, for U.S. Government purposes. 